\title{
Nuevo enfoque para la localización óptima de reconectadores en sistemas de distribución considerando la calidad del servicio y los costos de inversión
}

\author{
A new approach to optimal allocation of reclosers in distribution systems considering \\ service quality and fixed costs
}

Oscar D. Montoya G. ${ }^{1} \quad$ Ricardo A. Hincapié I. ${ }^{1} \quad$ Mauricio Granada E. ${ }^{1}$

Recibido 5 de noviembre de 2013, aceptado 4 de junio de 2015

Received: Noviembre 5, $2013 \quad$ Accepted: June 5, 2015

\begin{abstract}
RESUMEN
En este artículo se presenta una nueva metodología para la ubicación óptima de reconectadores en sistemas de distribución de energía eléctrica. La metodología considera en forma simultánea la instalación de reconectadores normalmente cerrados y abiertos, destinados al aislamiento de fallas y restauración del servicio, respectivamente. El problema es descrito por un modelo matemático multi-objetivo, donde la primera función objetivo minimiza el nivel de energía no servida del sistema (NENS) y la segunda los costos de inversión del proyecto. El conjunto de restricciones considera criterios operativos del sistema. Como estrategia de solución se introduce el concepto de áreas operativas, las cuales disminuyen esfuerzos computacionales al considerar que el NENS por área permanece constante. En la solución del problema se emplea un algoritmo elitista de ordenamiento no dominado (NSGA-II). Los resultados obtenidos reflejan la validez de la metodología propuesta y su posibilidad de aplicación a sistemas de distribución reales.
\end{abstract}

Palabras clave: Algoritmo NSGA-II, confiabilidad, optimización multi-objetivo reconectadores, sistemas de distribución.

\begin{abstract}
This article presents a new methodology for the optimal placement of reclosers on electric power distribution systems. The methodology simultaneously considers the installation of reclosers normally closed and open, in order to fault isolation and service restoration, respectively. The problem is described by a multiobjective mathematical model, where the first objective function minimizes the unserved energy level of the system (NENS) and the second one minimizes the investment costs of the project. The set of constraints considers operational criteria of the system. As a solution strategy the concept of operational areas is introduced, which reduce computational effort considering that the NENS by area remains constant. In the problem solution, a Non-dominated Sorting Genetic Algorithm II (NSGA-II) is employed. The results show the validity of the proposed methodology and its applicability to real distribution systems.
\end{abstract}

Keywords: NSGA-II Algorithm, reliability, multi-objective optimization, reclosers, distribution systems.

\section{INTRODUCCIÓN}

Los sistemas de distribución reciben la energía proveniente de los sistemas de transmisión y la entregan a los consumidores finales, con valores de tensión y frecuencia adecuados para el consumo. Para garantizar estas características ante eventos que alteren sus condiciones de operación normal, las empresas de distribución eléctrica emplean estrategias de detección y aislamiento de zonas

\footnotetext{
1 Programa de Ingeniería Eléctrica. Facultad de Ingenierías. Universidad Tecnológica de Pereira. Pereira, Colombia. E-mail: odmontoya@utp.edu.co; ricardohincapie@utp.edu.co; magra@utp.edu.co
} 
falladas y recuperación de zonas que quedan por fuera del servicio, para así disminuir los efectos causados por el evento y mejorar los indicadores de confiabilidad del sistema [1].

Adicionalmente los entes reguladores con el fin de garantizar a los usuarios un servicio de energía eléctrica seguro y confiable, fijan políticas de calidad y confiabilidad que son de obligatorio cumplimiento para las empresas distribuidoras [2]. Esto implica un gran reto para las empresas, ya que la regulación premia la buena calidad del servicio, pero castiga con sanciones económicas a quienes están fuera de sus lineamientos.

De acuerdo a lo anterior, las electrificadoras se han visto en la necesidad de automatizar sus redes eléctricas para mejorar las condiciones operativas de su sistema y cumplir con las políticas regulatorias del sector. Los reconectadores, debido a sus condiciones de operación automática, permiten dar cumplimiento a estas situaciones, dado que posibilitan el aislamiento de fallas y la recuperación de zonas de carga afectadas. Sin embargo, es necesario ubicar adecuadamente estos elementos sobre las redes, con el fin de obtener un máximo beneficio para su utilización. Debido a la gran cantidad de posibles ubicaciones y las diferentes características operativas y topológicas que presentan los sistemas de distribución, su ubicación presenta una gran complejidad, por lo que es importante contar con herramientas que permitan localizarlos adecuadamente.

Por lo tanto surge la necesidad de desarrollar estrategias de localización de reconectadores que garanticen el mejor beneficio posible en la operación de la red, el máximo beneficio económico y el cumplimiento de estándares regulatorios.

Dentro de este contexto, los reconectadores se presentan como una alternativa adecuada para minimizar los efectos producidos por las fallas en la operación normal del sistema. Dado que las fallas afectan principalmente la calidad del servicio, esta puede ser cuantificada calculando el NENS promedio resultante de un análisis de contingencias $n$-1. La ubicación de reconectadores normalmente abiertos (RNA) esencialmente tiene como objetivo la restauración del servicio a través de la transferencia automática de carga entre circuitos vecinos.
La ubicación de reconectadores normalmente cerrados (RNC) define las zonas que pueden ser aisladas en caso de una falla; por lo tanto, la combinación de ambas disposiciones determina la conformación de diferentes áreas operativas, dentro de las cuales la ocurrencia de una falla en cualquiera de sus elementos producirá un NENS constante. La localización de este tipo de dispositivos con el propósito de reducir el NENS del sistema es un problema combinatorial de alta complejidad matemática. Dado que la minimización de los índices de confiabilidad y calidad están sujetos a una disponibilidad máxima de recursos económicos por parte de las empresas prestadoras del servicio, es necesario abordar el problema desde una óptica multi-objetivo [3].

A diferencia de otros trabajos, en este artículo se presenta una metodología multi-objetivo que permite la ubicación óptima de RNA y RNC en forma simultánea, con el objetivo de mejorar la calidad del servicio considerando el costo de inversión y utilizando como indicador de sensibilidad el NENS. Estos dispositivos facilitan la interacción entre circuitos vecinos con el fin de tener un mayor grado de confiabilidad y un mayor margen de maniobrabilidad bajo ciertas circunstancias operativas. La complejidad computacional del problema es reducida introduciendo el concepto de áreas operativas con un NENS constante. Adicional a esto se consideran en el modelado del problema restricciones de tipo operativo y financiero, que son evaluadas a través de flujos de carga y un análisis económico, respectivamente. Para solucionar el problema se emplea como técnica de solución un algoritmo genético de ordenamiento no dominado (Nondominated Sorting Genetic Algorithm II NSGA II).

La organización de este documento es como sigue. En la sección 2 se presenta la descripción general del problema. En la sección 3 se describe la metodología propuesta. En la sección 4 se presenta la aplicación de la metodología y los resultados obtenidos. Finalmente se presentan las conclusiones derivadas de este proyecto.

\section{DESCRIPCIÓN GENERAL DEL PROBLEMA}

En esta sección se presenta la revisión del estado del arte y la formulación general para solucionar 
el problema de la ubicación óptima de RNA y RNC, tomando en consideración el NENS como indicador de confiabilidad, el costo de instalación de dispositivos como sensibilidad económica y las restricciones operativas asociadas al sistema de distribución [4].

Revisión del estado del arte

Para dar solución a este problema, esta temática ha sido abordada con diferentes formulaciones matemáticas y técnicas de solución, y presentando metodologías con gran desempeño y alto grado de aplicabilidad.

En [5] presentan una metodología para la localización óptima de reconectadores, seccionalizadores y fusibles en sistemas de distribución a través de un modelo matemático de programación no lineal entera mixta, cuya función objetivo minimiza el costo anual de la energía no servida. Utilizan como técnica de solución un algoritmo genético y para validar la metodología se emplea un sistema de distribución de dimensiones reales.

En [6] proponen una metodología para evaluar los indicadores de confiabilidad de un sistema de distribución (System Average Interruption Duration Index-SAIDI and System Average Interruption Frequency Index-SAIFI) considerando dispositivos de recierre automático a través de un algoritmo por enumeración de contingencias empleando modelos de Markov. Las pruebas fueron realizadas en un sistema de distribución brasileño.

En [7] plantean un método para localizar reconectadores y seccionalizadores de forma óptima en un sistema de distribución, para reducir la energía no servida (ENS) causada por la ocurrencia de fallas. El modelo considera las restricciones de capacidad de los elementos, así como la calidad de la potencia con la que se atienden las cargas. Utilizan una metodología en cascada, localizando inicialmente reconectadores y seccionalizadores y empleando un algoritmo eficiente de enumeración para determinar la topología final de la red. Luego deciden cuáles deben ser instalados en función de las limitaciones económicas. La metodología se valida empleando un sistema de distribución real.

En [8] proponen la localización óptima de reconectadores y seccionalizadores minimizando el costo total de inversión y el costo asociado a la ENS, el cual se calcula dependiendo del tipo de usuario; adicionalmente presentan una zonificación de los circuitos en función de los dispositivos de protección instalados. Usan como técnica de solución un algoritmo inmune y validan la propuesta en un sistema de distribución real.

En [9] emplean un modelo no lineal entero mixto para la localización óptima de dispositivos de protección sobre un sistema de distribución, considerando limitaciones técnicas y económicas, además de una clasificación de ramales por nivel de importancia; consideran como función objetivo la minimización de los costos de instalación de dispositivos y de interrupción del servicio ocasionado por las fallas. Usan como técnica de solución un algoritmo de búsqueda tabú reactivo y validan la propuesta en un sistema de características reales.

En [10] presentan una metodología para localización de dispositivos de protección a través de un modelo no lineal con una función objetivo denominada índice de restauración, la cual permite simplificar el problema; emplean como técnica de solución un algoritmo genético y evalúan el desempeño de la metodología con un sistema de distribución real.

En [11] desarrollan un modelo matemático para la minimización de los indicadores SAIDI y SAIFI, además del costo de inversión asociado a los elementos de protección; proponen el uso de reconectadores, switches y fusibles como un modelo de optimización mono-objetivo usando el programa de optimización comercial GAMS con el solver BARON, realizando una comparación con respuestas obtenidas mediante la implementación de algoritmos genéticos.

\section{Operación de reconectadores}

Los RNC son elementos de protección que tienen la capacidad de detectar e interrumpir momentáneamente en un sistema de distribución las corrientes de cortocircuito producidas por una falla; luego de su apertura y después de determinado tiempo el circuito es nuevamente energizado de forma automática [2, 12]. Si las condiciones de falla en el sistema han sido superadas satisfactoriamente, el sistema continuará operando de manera normal; de lo contrario, el reconectador repetirá las secuencias de apertura y cierre tantas veces como sea necesario 
para despejar la falla o hasta un límite preestablecido de operaciones. Es de notar que las operaciones del reconectador están gobernadas por diferentes intervalos de tiempo dados por curvas de operaciones rápidas y lentas [13].

Adicionalmente los RNA son usados como dispositivos de maniobra automática que permiten interconectar diferentes circuitos para reducir el impacto de las fallas en la calidad del servicio, a través de esquemas de reconfiguración automática. Esto permite mejorar los indicadores de confiabilidad y por lo tanto, en algunos esquemas regulatorios como en el caso colombiano, beneficios económicos para las empresas distribuidoras [14].

La operación conjunta de ambos elementos (RNA y RNC) permite seccionar un sistema de distribución en función de áreas operativas, cuya principal característica es delimitar las regiones de actuación y facilitar la maniobrabilidad y coordinación de los dispositivos de protección, para aislar las áreas afectadas por la falla y restaurar la mayor cantidad de carga posible a través de la transferencia de estas áreas hacia circuitos vecinos.
Con el fin de ilustrar un sistema de distribución y las posibles ubicaciones de los RNA y RNC, considerar la red de la Figura 1. Para este sistema las fuentes de generación alternas hacen parte de redes vecinas, cada una con circuitos radiales independientes (subestaciones 2, 3 y 4). Para efectos prácticos no se presentan en forma detallada; solo se muestran como posibles puntos de transferencia de carga en caso de presentarse una falla en alguna de las áreas conectadas a la subestación 1 .

Suponer que existe una falla en el área operativa 3 en cualquiera de los tramos contenidos en ella. Ante este evento el sistema de protección cambia el estado operativo de los RNC R3, R4 y R5 de normalmente cerrados a abiertos, aislando así la sección bajo falla. En esta situación las áreas operativas 4,5 y 6 quedan sin servicio.

Con el fin de restaurar la mayor cantidad de carga, los RNA cambian su estado operativo de abiertos a cerrados para permitir la transferencia de carga de un circuito a otro. De acuerdo a esto, al abrir el RNC que interconecta las áreas 4 y 6 (R6), y al abrir los RNA R8, R9 y R10, las áreas operativas 4, 5 y

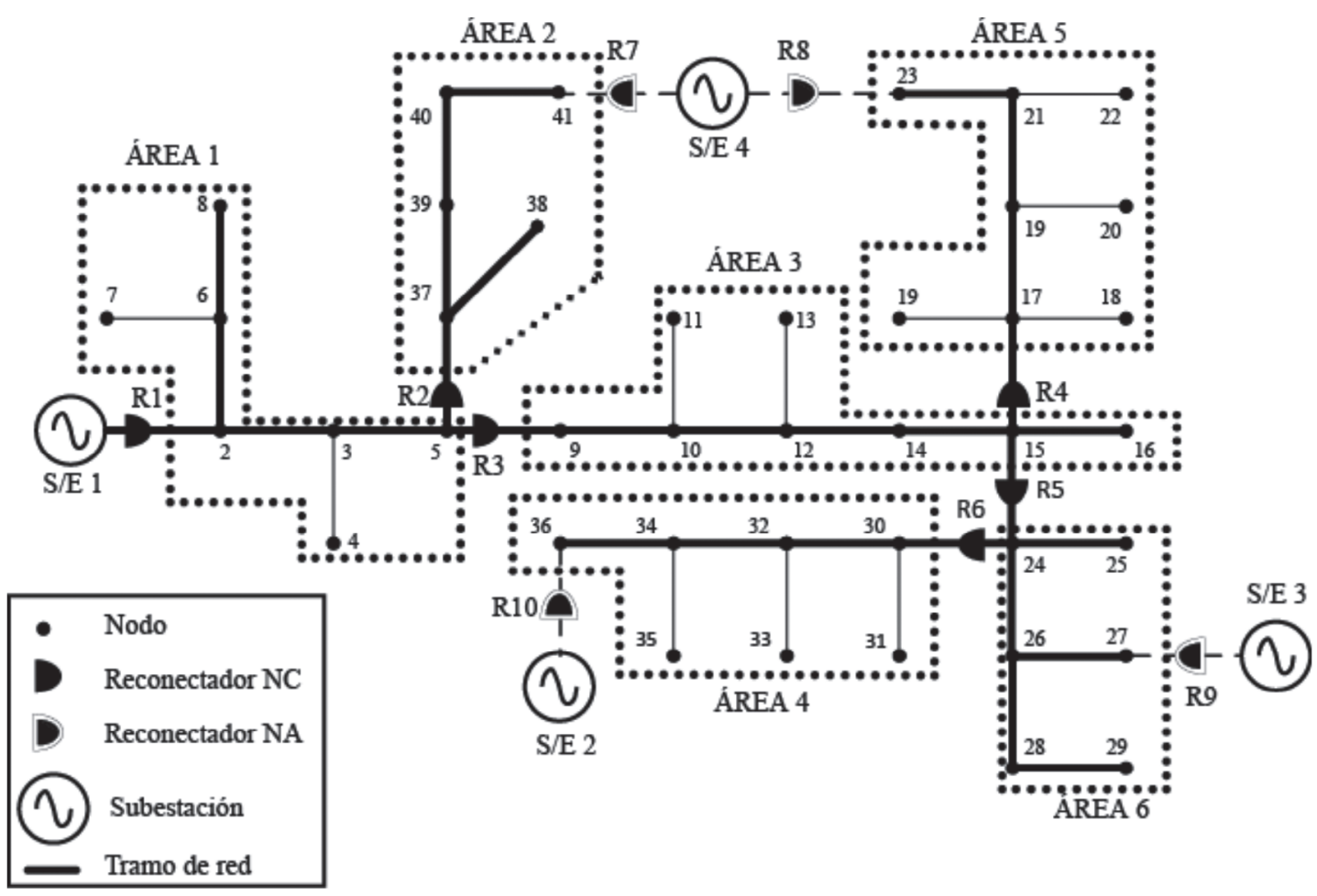

Figura 1. Sistema antes de la falla. 
6 quedan alimentadas a través de las subestaciones 2,4 y 3 , respectivamente.

Cuando se realizan estas maniobras es importante verificar el cumplimiento de las cargabilidades de los elementos y los límites de tensión, por lo que es necesario emplear flujos de carga para obtener las características operativas del sistema bajo estas circunstancias. La nueva configuración del sistema se ilustra en la Figura 2.

\section{Indicadores de Confiabilidad}

Para una empresa de distribución de energía eléctrica es de gran importancia entregar un servicio de calidad a sus usuarios, para lo cual los entes reguladores exigen cumplimiento de índices de confiabilidad, que de una u otra manera cuantifican la calidad del servicio [11]. Existen diferentes indicadores de confiabilidad como SAIFI, SAIDI, índice de frecuencia de interrupción media de los usuarios (CAIFI) y el índice de duración de interrupción media de los usuarios (CAIDI), entre otros [15].

Adicionalmente existe un indicador denominado energía no servida (ENS), que permite cuantificar el efecto producido por una falla en cada uno de los componentes de la red, determinando la cantidad promedio de energía que se deja de suministrar [6].

En forma general la ENS es una métrica del impacto que produce sobre el sistema la ocurrencia de contingencias que alteran las condiciones topológicas de la red [6]. Matemáticamente se expresa de acuerdo a la ecuación (1):

$$
E N S=\sum_{i=1}^{n_{i}} \sum_{j=1}^{n_{j}} \sum_{k=1}^{n_{k}} L_{i k} \times r_{j} \times \lambda_{j}
$$

Donde,

$n_{k} \quad$ número de puntos de carga aislados durante una contingencia.

$n_{j} \quad$ número de eventos de falla.

$n_{i} \quad$ cantidad de pasos considerados de la curva de carga.

$\lambda_{i} \quad$ tasa de fallas en el punto de carga i.

$r_{j} \quad$ tiempo promedio de recuperación de la falla para la contingencia $j$.

$L_{i k}$ potencia demandada en el punto de carga $i$ en el paso $k$ de la curva de carga.

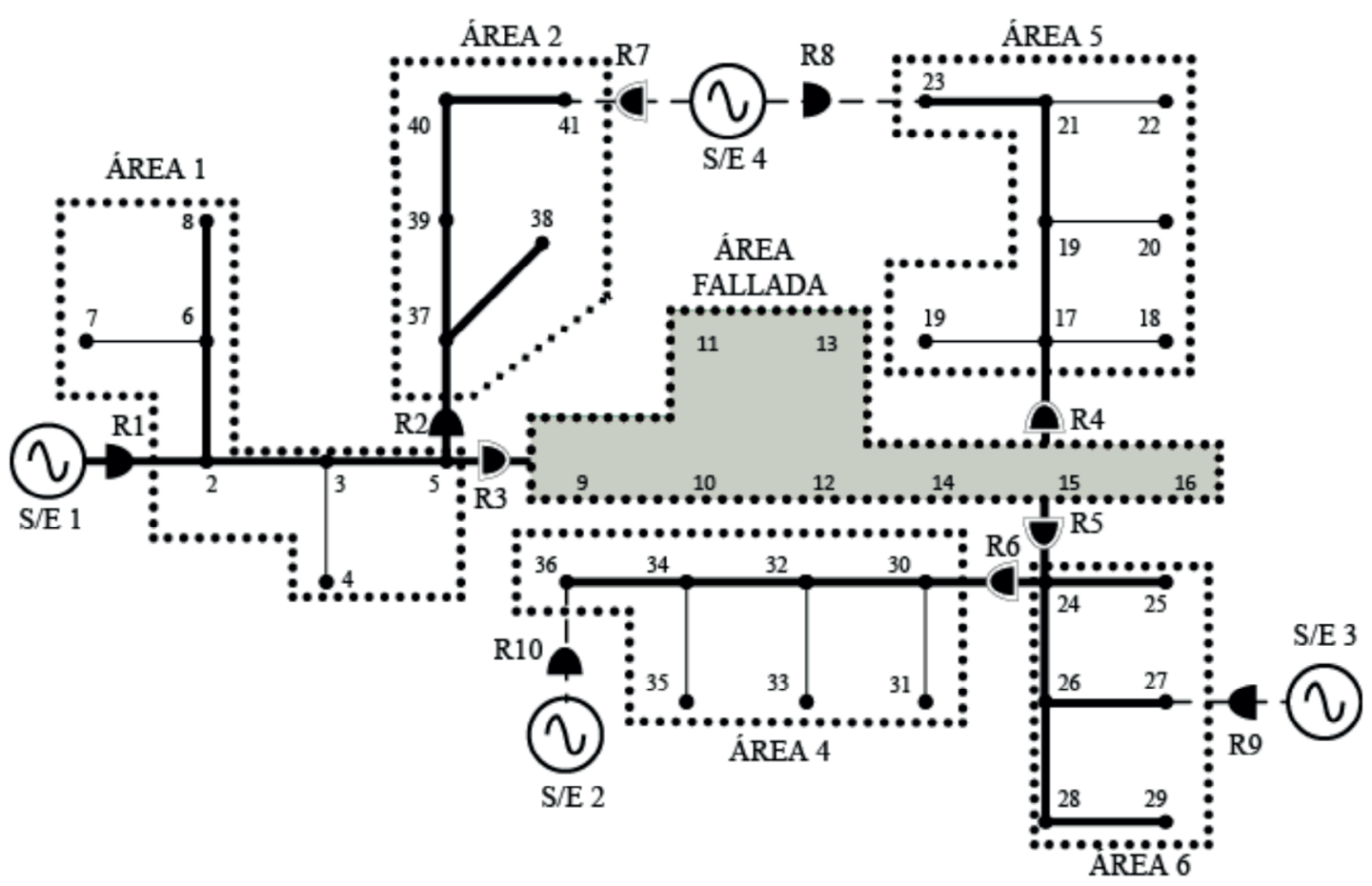

Figura 2. Sistema después de la falla. 
Esta formulación presenta el cálculo de la energía no servida para un sistema de distribución a través de un proceso de evaluación de contingencias sucesivas [11], de forma que se agrega la energía que no puede ser atendida en cada punto de concentración de carga, hasta que se han evaluado contingencias en todo el sistema (criterio $n-1$ ). Adicional a esto se incluye en el cálculo la curva de carga para modelar con mayor precisión el comportamiento real de la red de distribución [16].

Para la evaluación de la energía no servida en presencia de reconectadores, la metodología propuesta en este artículo se basa en la aplicación de un análisis por áreas o regiones resultantes de la operación conjunta entre RNA y RNC. Así, la evaluación de la ecuación (1) en un contexto de múltiples áreas, requiere el conocimiento previo de la ubicación y del estado de cada reconectador, para posteriormente, ejecutar un algoritmo especializado que permita cuantificar el nivel de energía no servida. Las particularidades de este criterio multiárea son presentadas en una sección posterior.

\section{Criterio multiárea}

La operación conjunta de RNA y RNC permite seccionar un sistema de distribución en función de áreas operativas. Cuando se presenta una falla en cualquier tramo de red de un área operativa, el valor de ENS es el mismo, sin importar en qué lugar dentro del área ocurre la falla.

De acuerdo a esto, al considerar en forma simultánea la ubicación de estos elementos, se pueden encontrar áreas operativas que permitan mejorar las condiciones operativas bajo ciertas características del sistema. Adicionalmente cuando se consideran áreas operativas disminuye la complejidad del problema, debido a la disminución en el esfuerzo computacional asociado a la evaluación del criterio de contingencias $n-1$, ya que se reduce la evaluación de los flujos de carga.

A diferencia de otros trabajos, en este artículo se considera la ENS constante en función de las áreas operativas. Este valor es usado como indicador de sensibilidad para la localización de estos elementos, por lo que en este trabajo, y con el fin de diferenciarlo del indicador de ENS clásico, este valor es denominado nivel de energía no servida (NENS).
Su diferencia fundamental con (1) radica en la forma de determinar el nivel de energía no servida en función de las áreas operativas bajo el criterio de energía constante, de forma que esta se calcula por áreas operativas y no por tramos de red.

\section{Optimización multi-objetivo}

Un problema de optimización multi-objetivo requiere utilizar simultáneamente un cierto número de objetivos con diferentes soluciones óptimas individuales, donde un objetivo no puede ser mejorado sin la degradación de otro [17]. Debido a esto, en lugar de una única solución óptima del problema, se encuentran un conjunto de soluciones óptimas denominadas frente o frontera de Pareto [18].

La forma canónica que representa las condiciones de un problema de optimización multi-objetivo es:

$$
\begin{aligned}
\max / \min = & f_{m}(x), m=1,2, \ldots, M \\
\text { s.a. } & g_{j}(x) \geq 0, j=1,2, \ldots, J \\
& h_{k}(x) \geq 0, k=1,2, \ldots, K \\
& x_{i}^{\text {min }} \leq x_{i} \leq x_{i}^{\max }, i=1,2, \ldots, I
\end{aligned}
$$

Donde $x$ es el vector de decisión y $f_{m}(x)$ corresponde a cada una de las $m$ funciones objetivo del problema.

El conjunto de soluciones factibles $S^{*}$ del modelo descrito en (2) se define como las variables de decisión que satisfacen en el punto óptimo las restricciones de igualdad definidas por $h_{k}(x)$ y de desigualdad $g_{j}(x)$, es decir:

$$
S^{*}=\{\bar{x} \in \bar{S} / g(x) \geq 0 \wedge h(x)=0\}
$$

Cualquier punto $x$ en $S^{*}$ define una solución factible. Realizando un mapeo desde el espacio de soluciones factibles dado por $S^{*}$ al espacio de los objetivos a través de una transformación lineal, se define el espacio de los objetivos como $Z^{*}$ y puede ser representado matemáticamente como:

$$
Z^{*}=\left\{\left[f_{1}(x), f_{2}(x), \ldots, f_{m}(x)\right], x \in S^{*}\right\}
$$

\section{Modelo matemático considerando áreas operativas}

Dado que al mejorar la confiabilidad del sistema (disminución de NENS) se aumentan los costos de 
inversión debido al requerimiento de un número mayor de reconectadores, este problema es tratado en este artículo de forma multi-objetivo, ya que no existe una correlación directa entre el comportamiento de las dos funciones objetivo.

De acuerdo a esto el problema de localización óptima de RNA y RNC en sistemas de distribución, puede ser formulado a través de un modelo de optimización multi-objetivo descrito a continuación.

$$
\begin{aligned}
\min N E N S & =\sum_{y \in \Omega_{y}}\left[\left(\sum_{k \in \Omega_{y N}} P_{k, y}\right)\left(\sum_{i j \in \Omega_{y, L}} \lambda_{i j, y} * D_{i j, y} * r_{i j, y}\right)\right] \\
\operatorname{mín} C_{f} & =\sum_{m \in \Omega_{D}} C_{m} * x_{m}
\end{aligned}
$$

s.a.

$$
\begin{aligned}
& \left|I_{i j, y}\right| \leq I_{i j, y}^{m a ́ x} \\
& \forall i j \in \Omega_{y L} \\
& \forall y \in \Omega_{y} \\
& V_{k, y}^{\min } \leq V_{k, y} \leq V_{k, y}^{\operatorname{má} x} \\
& \forall k \in \Omega_{y N} \\
& \forall y \in \Omega_{y} \\
& E Q^{u}\left(P_{k, y}^{D}, Q_{k, y}^{D}, V_{k, y}, \theta_{k, y}\right)=0 \quad \begin{array}{l}
\forall k \in \Omega_{y N} \\
\forall y \in \Omega_{y}
\end{array} \\
& C T R_{i j, y} \geq \sum_{k \in \Omega_{y N}} S_{k, y}^{D} \quad \forall i j \in \Omega_{y L} \\
& \sum_{m \in \Omega_{D}} x_{m} \leq N D_{\text {máx }} \\
& \sum_{m \in \Omega_{D}} C_{m} * x_{m} \leq R F_{\text {máx }} \\
& C R^{u}\left(x_{m}, k, y\right)=0
\end{aligned}
$$

Donde la nomenclatura empleada para interpretar el modelo multi-objetivo es:

Conjuntos:

$\Omega_{y} \quad$ conjunto que contiene las áreas que se conforman por la ubicación de los RNA y RNC.

$\Omega_{y N} \quad$ conjunto de todos los nodos que pertenecen al área $y$.

$\Omega_{y L} \quad$ conjunto de todos los tramos de red que pertenecen al área $y$.
$\Omega_{D} \quad$ conjunto de todos los puntos candidatos a la ubicación de un reconectador $x_{m}$.

Índices:

$k \quad$ índice que recorre todos los nodos.

ij índice que recorre todos los tramos de red.

$m \quad$ índice que recorre todos los puntos candidatos a la ubicación de reconectadores.

y índice que recorre todas las áreas.

Funciones:

$C R^{u}(*)$ función que determina que todas las configuraciones cumplen la condición de radialidad.

$C f \quad$ costos fijos de inversión.

$E Q^{u}\left(^{*}\right)$ función que garantiza el balance de potencia en cada nodo $k$ del sistema

NENS nivel de energía no servida.

Parámetros:

$\lambda i j, y \quad$ tasa de fallas permanentes en el tramo $i j$ perteneciente al área $y$.

$D_{i j, y} \quad$ longitud del tramo de red $i j$ perteneciente al área $y$.

$r_{i j, y} \quad$ tiempo promedio de reparación de la falla ocurrida en tramo $i j$ perteneciente al área $y$.

$C T R_{i j, y}$ capacidad de transferencia del reconectador instalado en el tramo de red $i j$ perteneciente al área $y$.

$R F_{\text {máx }}$ máximo recurso disponible para invertir en el proyecto.

$N D_{\text {máx }}$ máximo número de dispositivos de protección disponibles

$C_{m} \quad$ costo de instalación del reconectador $m$.

$I_{i j, y} \quad$ corriente que circula por el tramo de red $i j$ perteneciente al área $y$.

$I_{i j, y}^{m a ́ x} \quad$ máxima corriente que soporta el conductor del tramo de red $i j$.

$V_{k, y}^{\min } \quad$ voltaje mínimo permitido en el nodo $k$ perteneciente al área $y$.

$V_{k, y}^{m a ́ x} \quad$ voltaje máximo permitido en el nodo $k$ perteneciente al área $y$.

Variables binarias:

$x_{m} \quad$ variable binaria de decisión de instalación del reconectador $m$. 
Variables continuas:

$V_{k, y} \quad$ voltaje en el nodo $k$ perteneciente al área $y$.

$S_{k, y}^{D} \quad$ demanda de potencia aparente en el nodo $k$ perteneciente al área $y$.

$Q_{k, y}^{D} \quad$ demanda de potencia aparente en el nodo $k$ perteneciente al área $y$.

$P_{k, y}^{D} \quad$ demanda de potencia activa en el nodo $k$ perteneciente al área $y$.

$\theta_{k, y} \quad$ ángulo del voltaje en el nodo $k$ perteneciente al área $y$.

En forma general, la formulación matemática descrita anteriormente por las ecuaciones (5) a (11), busca la minimización del indicador de confiabilidad denominado NENS dado por (5) y del costo fijo o de inversión presentado en (6), a través de una estrategia de selección de elementos de protección que cumpla con un conjunto de restricciones técnicas y operativas.

La ecuación (5) permite la determinación del NENS para una red de distribución en función de la ubicación de los RNC y RNA, para detectar y aislar la parte del circuito de distribución que se encuentre bajo la influencia de una falla. Por otro lado (6) cuantifica el costo de los reconectadores y las obras civiles necesarias para su ubicación y puesta en funcionamiento.

El conjunto de restricciones para la localización óptima de estos elementos está dado por las ecuaciones (7) a (12). La ecuación (7) determina que no se presenten tramos de red sobrecargados. Con la restricción (8) se garantiza que las tensiones en los nodos del sistema se encuentren dentro de los rangos permitidos por normatividad. La expresión (9) garantiza que se cumple el balance de potencia para todos los nodos del sistema. La ecuación (10) asegura que la capacidad de transferencia de potencia a través de un RNA tiene que ser superior a la cantidad de carga conectada aguas abajo de él, es decir, a la suma de todas las demandas conectadas a los $k$ nodos contenidos en el área $y$. Las ecuaciones (11) y (12) corresponden a la cantidad de reconectadores a ser localizados en el sistema y las limitaciones de inversión, respectivamente. Finalmente la expresión (13) representa una función que garantiza la radialidad del sistema para cada configuración de RNA y RNC propuesta y del número de áreas que se conformen.

\section{METODOLOGÍA PROPUESTA}

En esta sección se describen los aspectos más relevantes sobre la metodología propuesta para ubicar en forma simultánea reconectadores normalmente abiertos y cerrados, con el fin de mejorar los indicadores de confiabilidad del sistema (NENS) al menor costo posible.

\section{Codificación del problema}

La codificación del problema está dada por un vector de variables binarias, de tamaño igual al número de reconectadores normalmente cerrados $(n)$ y abiertos $(m)$ que se pueden instalar en el sistema, donde cada reconectador tiene asociada una posición en el vector. Cuando la variable binaria vale uno, quiere decir que se propone la instalación de dicho reconectador; en caso contrario vale cero. En la Figura 3 se presenta la codificación propuesta.

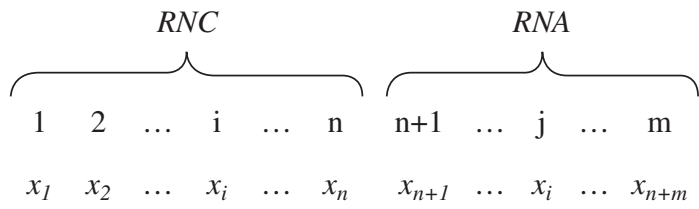

Figura 3. Esquema de codificación propuesto.

Con el fin de explicar el esquema empleado, considerar el sistema de distribución de la Figura 4a en el cual se tienen propuestos cinco reconectadores normalmente cerrados y seis reconectadores normalmente abiertos. Bajo esta situación el vector tendrá once posiciones que describen cada uno de los elementos considerados.

Asumiendo que se propone la instalación de los RNC 2, 4 y 5, y los RNA 7, 8 y 10, se obtiene la configuración presentada en la Figura 4b. El vector codificación que describe esta solución se presenta en la Figura 5.

\section{Evaluación de las condiciones operativas}

Para evaluar las contingencias sobre la red se usa un algoritmo de enumeración de eventos de falla que permite recorrer la red de distribución y determinar la cantidad de energía no servida para cada evento, realizando un acumulado general con todas las posibilidades de falla para la red.

Con el fin de evaluar las condiciones operativas de la red de distribución y las restricciones de regulación 


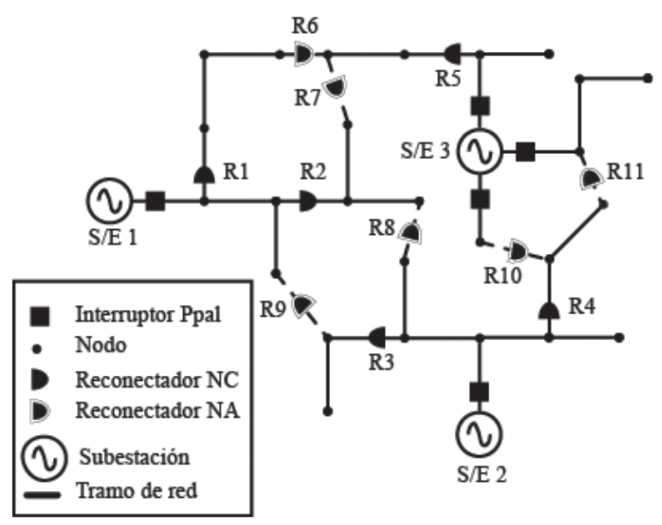

(a)

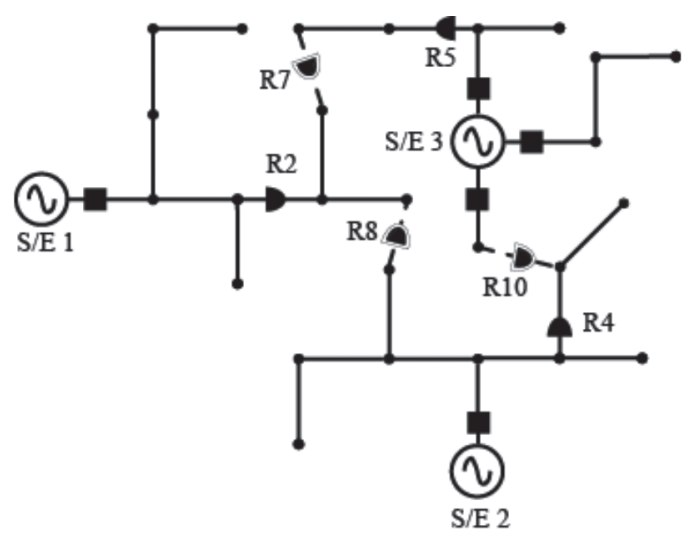

(b)

Figura 4. Ubicación de RNA y RNC.

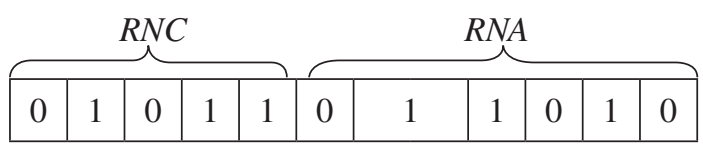

Figura 5. Codificación de la Figura 4b.

de tensión y máximo flujo de corriente por los conductores, se emplea un algoritmo de flujo de potencia de barrido iterativo [21], el cual permite establecer cada punto de operación que puede llegar a tener la red en función del cambio de topologías producto de una falla en el sistema y el posterior esquema de restauración de las áreas operativas.

\footnotetext{
Algoritmo NSGA II

Para resolver el problema de la ubicación de reconectadores en sistemas de distribución se emplea el algoritmo de optimización multi-objetivo NSGA II, propuesto por Deb [17]. Esta técnica es atractiva porque presenta dos características fundamentales: en primer lugar garantiza diversidad poblacional
}

durante el proceso de solución y en segundo lugar es un algoritmo elitista, lo que quiere decir que sólo son consideradas durante el proceso las mejores soluciones que se han encontrado en los diferentes frentes de Pareto.

La idea básica de este algoritmo es que durante el paso evolutivo $t$, el conjunto de descendientes $Q_{t}$ de tamaño $N$ se genera a partir del conjunto de padres $P_{t}$ del mismo tamaño, a partir de la aplicación de los operadores genéticos de selección, recombinación y mutación [19]. En función de las dos poblaciones anteriores $\left(P_{t}, Q_{t}\right)$ se forma una población $R_{t}$ de tamaño $2 N$. Después mediante un ordenamiento se clasifica la población en frentes de Pareto, lo cual consiste en determinar para cada individuo un nivel de dominancia frente a las demás soluciones; de acuerdo con esto sólo las mejores $N$ soluciones harán parte de la población $P_{t+1}$, en donde el proceso vuelve a comenzar [20].

Este proceso se repite hasta que transcurran un número de ciclos generacionales sin evolución o hasta que se alcanza un número predefinido de iteraciones [18].

La ventaja de emplear esta metodología es que no se obtiene una única solución, sino un conjunto de soluciones óptimas dadas por el frente de Pareto. Esto permite tener una gran cantidad de posibilidades de solución, las cuales pueden ser determinadas de acuerdo a los criterios específicos de cada empresa.

En la Figura 6 se presenta un diagrama de bloques donde se describe la metodología propuesta.

\section{APLICACIÓN Y RESULTADOS}

Para verificar la efectividad de la metodología propuesta se considera el sistema de distribución empleado en [22] (Figura 7), donde las líneas punteadas están asociadas a RNA y las líneas continuas a RNC. Cada circuito tiene una asociada una protección primaria a la salida de la subestación (cuadrados negros).

En las Tablas 1 y 2 se presenta la información asociada a los RNC y RNA, respectivamente. Los datos asociados a la carga conectada en cada nodo, tramos de red, tasas de falla y tiempos de reparación, están disponibles con los autores. En 


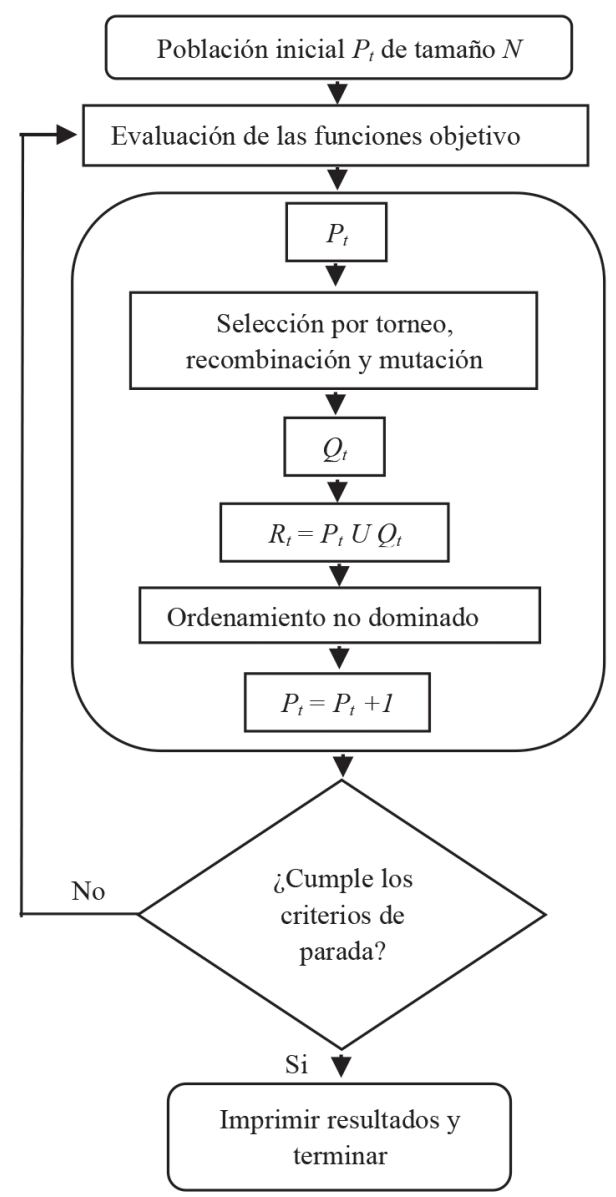

Figura 6. Diagrama de bloques de la metodología.

la Tabla 3 se presentan los parámetros empleados por el algoritmo.

La metodología propuesta se implementó en un computador Intel Core 2 Duo con 4GB de RAM y sistema operativo Windows 7 Professional mediante el software MATLAB 2010a.

El frente óptimo de Pareto obtenido al aplicar la metodología propuesta, se presenta en la Figura 8. En esta figura se observa la evolución de la población inicial (encerrada por la línea punteada) hacia el mínimo de las dos funciones objetivo, evidenciando su diversificación en el proceso evolutivo. Se nota como al inicio del proceso todas las alternativas tienen un costo elevado y con bajos niveles de energía no servida, y a medida que avanzan los ciclos generacionales algunos individuos de la población evolucionan hacia regiones de mejor calidad (puntos en la parte izquierda de la Figura).
Tabla 1. Características de RNC.

\begin{tabular}{|c|c|c|c|}
\hline Envío & Recibo & Codificación & Costo (US\$) \\
\hline 1 & 12 & R1 & 16.500 \\
\hline 2 & 9 & R2 & 16.500 \\
\hline 9 & 87 & R3 & 16.500 \\
\hline 19 & 50 & R4 & 16.500 \\
\hline 19 & 43 & R5 & 16.500 \\
\hline 20 & 46 & R6 & 16.500 \\
\hline 20 & 71 & R7 & 16.500 \\
\hline 32 & 83 & R8 & 16.500 \\
\hline 58 & 69 & R9 & 16.500 \\
\hline 66 & 67 & R10 & 16.500 \\
\hline 72 & 76 & R11 & 16.500 \\
\hline 93 & 97 & R12 & 16.500 \\
\hline 93 & 112 & R13 & 16.500 \\
\hline 93 & 118 & R14 & 16.500 \\
\hline 128 & 150 & R15 & 16.500 \\
\hline 128 & 140 & R16 & 16.500 \\
\hline 136 & 155 & R17 & 16.500 \\
\hline 158 & 181 & R18 & 16.500 \\
\hline 158 & 183 & R19 & 16.500 \\
\hline 158 & 160 & R20 & 16.500 \\
\hline 158 & 198 & R21 & 16.500 \\
\hline
\end{tabular}

Tabla 2. Características de RNA.

\begin{tabular}{|c|c|c|c|}
\hline Envío & Recibo & Codificación & Costo (US\$) \\
\hline 13 & 119 & R22 & 68.010 \\
\hline 18 & 96 & R23 & 38.605 \\
\hline 43 & 92 & R24 & 34.195 \\
\hline 53 & 23 & R25 & 25.250 \\
\hline 162 & 80 & R26 & 60.900 \\
\hline 191 & 20 & R27 & 86.610 \\
\hline 70 & 133 & R28 & 66.570 \\
\hline 82 & 141 & R29 & 27.185 \\
\hline 89 & 139 & R30 & 105.760 \\
\hline
\end{tabular}

Tabla 3. Parámetros empleados por NSGA-II.

\begin{tabular}{|c|c|}
\hline \multicolumn{2}{|c|}{ Parámetros } \\
\hline Tasa de mutación (\%) & 80 \\
\hline Tasa de recombinación (\%) & 90 \\
\hline Puntos de cruce & 1 \\
\hline Tiempo de ejecución & 281,49 \\
\hline Tamaño de la población & 20 \\
\hline Ciclos generacionales & 80 \\
\hline
\end{tabular}




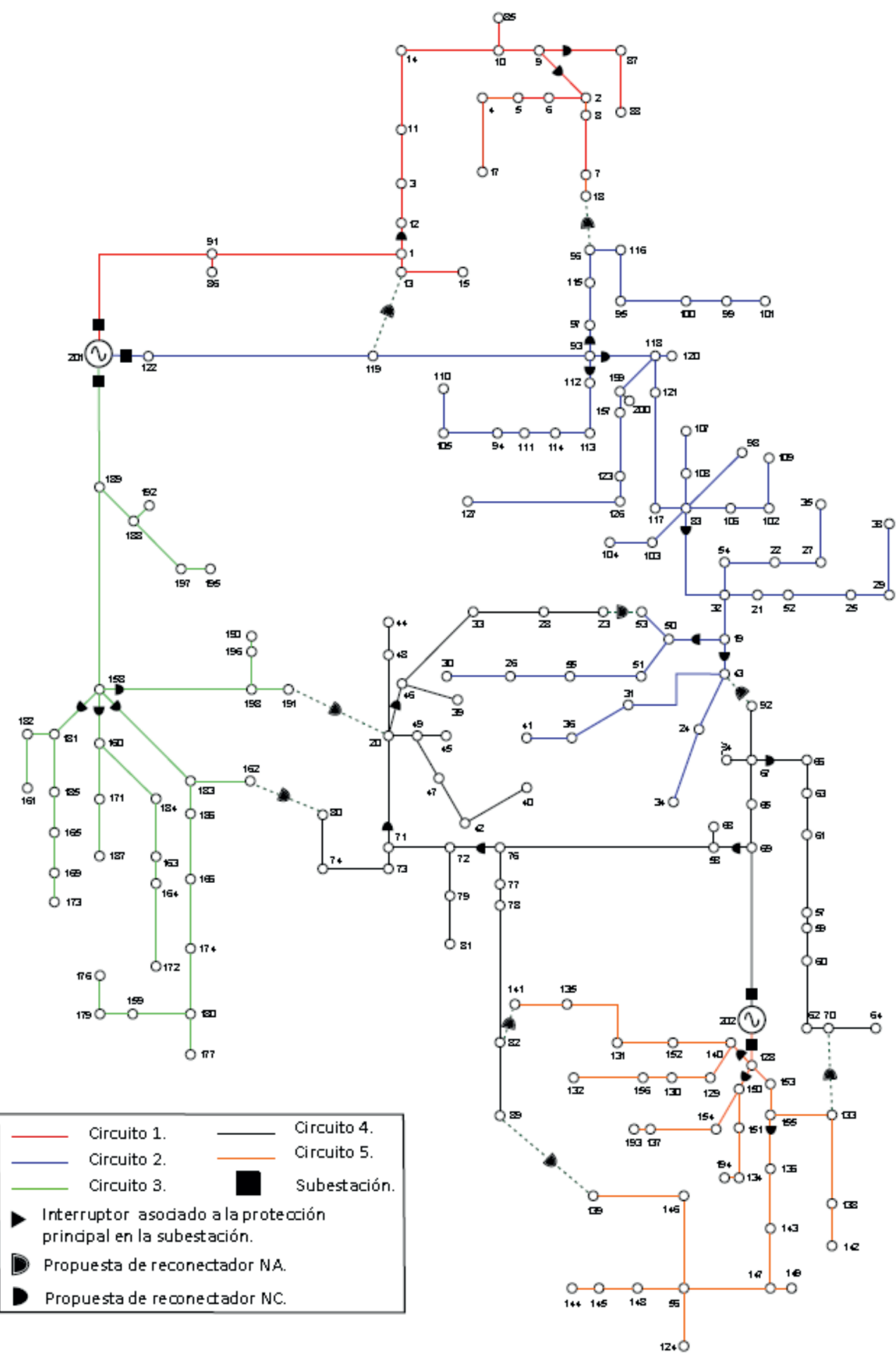

Figura 7. Sistema de prueba. 


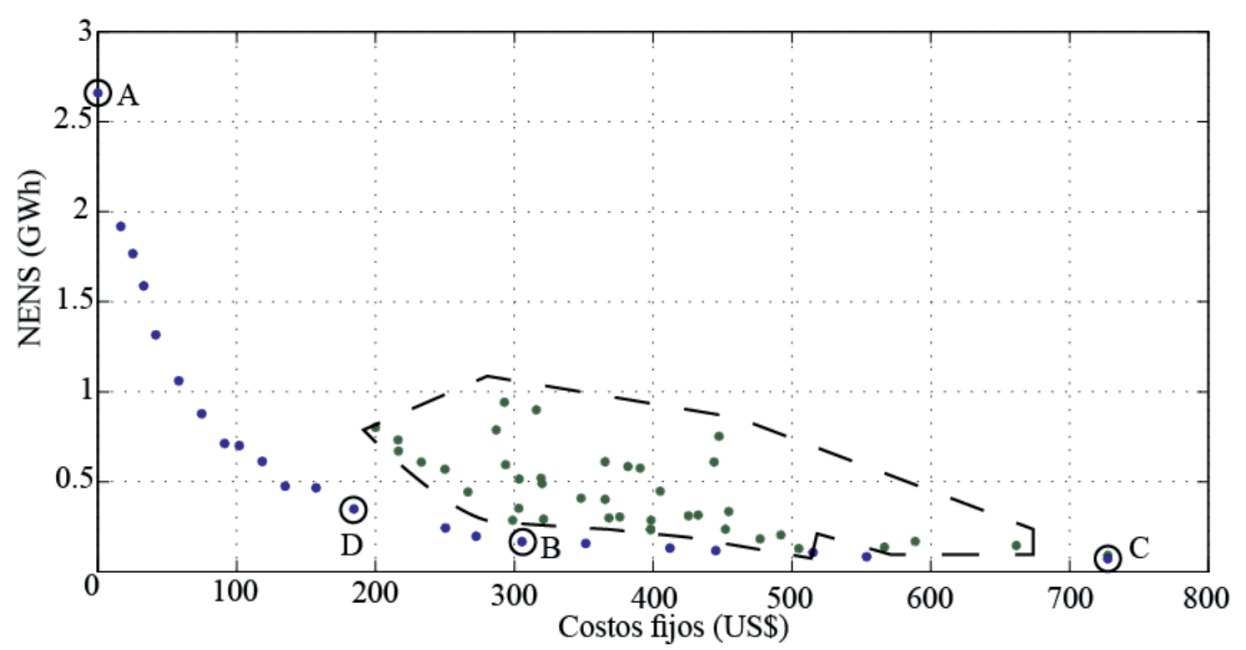

Figura 8. Frontera óptima de Pareto.

Se observa que existe una diferencia considerable entre la mejor y la peor solución para cada uno de los objetivos (puntos A y C), siendo entonces los rangos del nivel de energía no servida y del costo de inversión igual a 2,5876 GW-h y US\$727.585, respectivamente.

Cuando la inversión en reconectadores es mayor a US $\$ 300.000$, los individuos del frente no presentan mejoras significativas en la reducción del NENS. Por ejemplo, las soluciones de los puntos B y C tienen un costo de US\$305.540 y US\$727.585, respectivamente, y un valor de NENS de 167,5 MW-h y 72,5 MW-h. De acuerdo a esto, para reducir 95 MW-h se tendría que dar adicional un valor de US $\$ 422.045$; en otras palabras, para reducir un $3,5 \%$ de NENS (con respecto al mayor valor), se pagaría un 58\% adicional (con respecto al mayor valor), por lo que la relación costo/beneficio para la empresa de distribución no es tan representativa en este caso.

Dado que en los problemas multi-objetivo no se tiene una única solución óptima, sino un conjunto de soluciones óptimas dado por el frente de Pareto, la selección de la mejor configuración depende de los criterios particulares de cada empresa (operación de la red, limitaciones económicas, aplicación de prácticas operativas, etc.). Sin embargo en la literatura existen diversas técnicas (métricas) para seleccionar la mejor configuración [17].
Con el fin de ejemplarizar una posible solución al problema de localización óptima de RNA y RNC en el sistema de distribución empleado, se presenta en la Figura 9 la configuración orrespondiente al punto D de la Figura 8. Esta solución tiene un nivel de energía no servida igual a 349,4 MW-h con una inversión de US\$184.435, la cual propone la instalación de los RNC 2, 6, 8, 16, 17, 18 y 20, y los RNA 4 y 8.

\section{CONCLUSIONES}

Se desarrolló una metodología simple y eficiente que permite determinar la localización óptima de reconectadores en sistemas de distribución de energía eléctrica para mejorar el nivel de energía no servida, usando como técnica de solución un algoritmo NSGA II, obteniéndose un conjunto de soluciones óptimas no dominadas del frente óptimo de Pareto.

Se propuso una metodología novedosa para trabajar la ubicación óptima de RNC y RNA en forma conjunta para sistemas de distribución a través de un manejo en función de áreas operativas conformadas por el conjunto de dispositivos de protección aguas arriba y aguas abajo de un tramo de red, lo que permite agilizar los tiempos computacionales, ya que la energía no servida en estas áreas permanece constate para cualquier falla de tipo permanente que se presente en un tramo contenido en ella. 


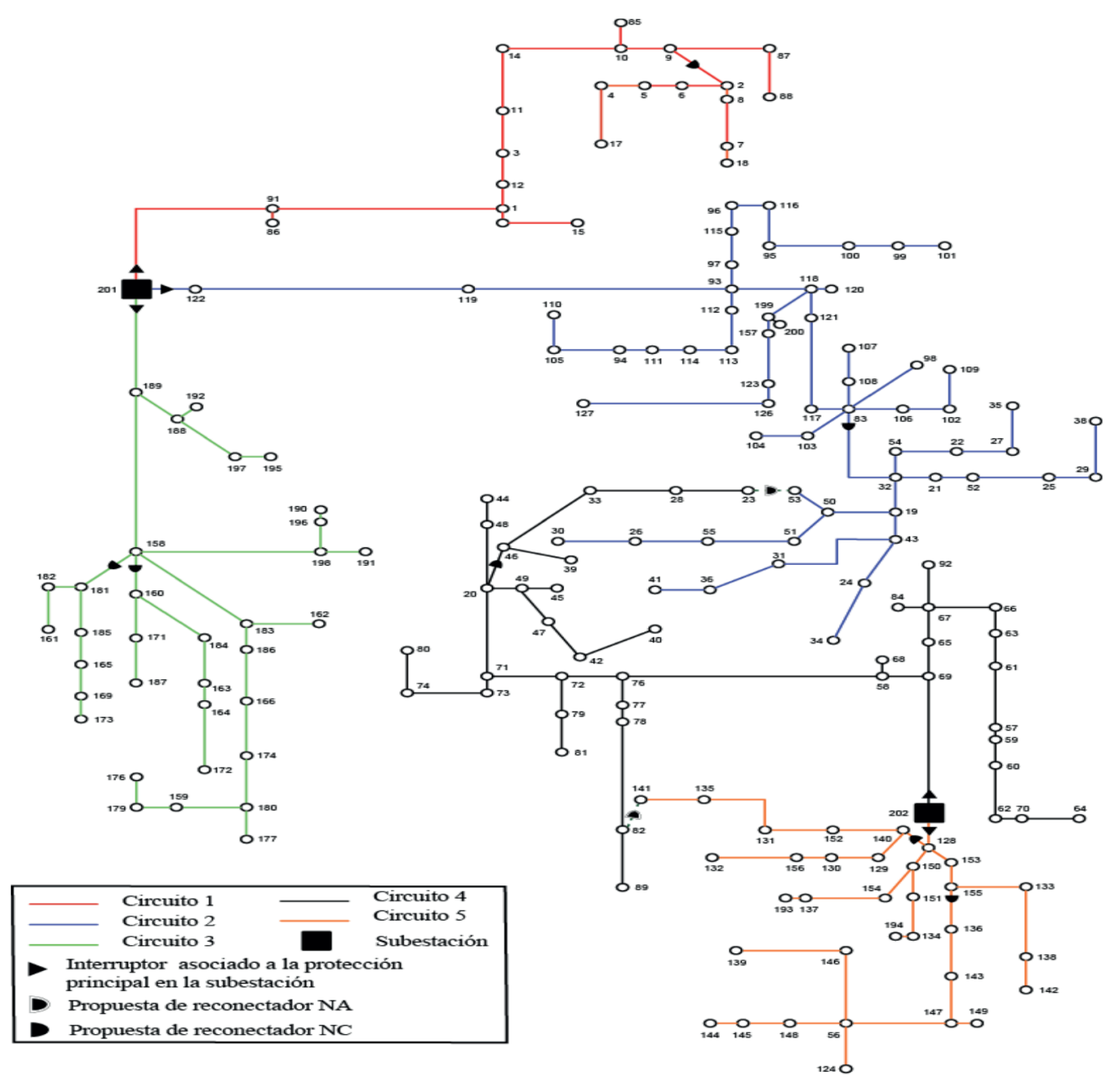

Figura 9. Configuración seleccionada.

Para evaluar el NENS en sistemas de distribución a través del criterio de contingencias $(n-1)$, es necesario la inclusión de flujos de carga que permitan evaluar las condiciones operativas de la red ante el cambio de topologías producto del proceso de aislamiento de fallas y restauración del servicio a través de reconexión con circuitos vecinos, hecho que evidencia la fortaleza del trabajo, proponiendo así un acercamiento al comportamiento real de los sistemas de distribución.

Lo propuesto en este trabajo permite obtener beneficios económicos para las empresas de distribución, dados por el mejoramiento en la continuidad del servicio debido a la reducción de energía no servida, incentivos recibidos por organismos de regulación y control producto del cumplimiento de índices de confiabilidad y un aumento del esquema tarifario ocasionado por el incremento en el valor del cargo por uso (para países con esquemas tarifarios como el colombiano).

Esta metodología puede ser integrada en redes de distribución bajo el concepto de smart grids, ya que puede trabajar en conjunto con otros dispositivos de protección mejorando la operación del sistema a partir de la automatización de las redes.

La metodología requirió de la adaptación de un sistema de prueba típico de procesos relacionados con planes de expansión del sistema de distribución de energía, con el fin de generar una base de datos que permitiese interrelacionar diferentes redes radiales de distribución y así generar caminos alternativos 
para suplencia de cargas afectadas indirectamente por las fallas. Esto quiere decir que se propone un nuevo sistema de prueba para trabajar en localización óptima de elementos de protección, lo que puede ser de gran utilidad para las líneas de investigación relacionadas con el sector eléctrico.

\section{AGRADECIMIENTOS}

Al programa de Jóvenes Investigadores e Innovadores de Colciencias del año 2012, por su apoyo a la propuesta de investigación presentada bajo el título "Localización Óptima de Reconectadores, Seccionalizadores y Fusibles en Sistemas de Distribución de Energía para Aislamiento de Fallas".

\section{REFERENCIAS}

[1] C.A. Peñuela and J. R.S. Mantovani, "Improving the grid operation and reliability cost of distribution systems with dispersed generation". IEEE Transactions on Power Systems. Vol. 29, pp. 2485-2496. August, 2013. ISSN: 0885-8950. DOI: $10.1109 /$ TPWRS.2012.2235863.

[2] H. Zhenga, Y. Chenga, B. Goub, D. Frankc, A. Bernc and W. Mustonc. "Impact of automatic switches on power distribution system reliability". Electric Power Systems Research. Vol. 83, pp. 51-57. February, 2011. ISSN: 0378-7796. DOI: 10.1016/j. epsr.2011.08.018

[3] G. Ferreira and A. Bretas. "A nonlinear binary programming model for electric distribution systems reliability optimization". Electrical Power and Energy Systems. Vol. 43, pp. 384392. December, 2012. ISSN: 0142-0615. DOI: 10.1016/j.ijepes.2012.05.070.

[4] C.A. Pulgarín, M.A. Rios, C.A. Acosta, R.A. Hincapié, M. Granada and R.A. Gallego. "Localización óptima de reconectadores normalmente abiertos para transferencia de carga". Mundo Eléctrico. Vol, 50. pp. 38-44. Marzo, 2013. ISSN: $1692-7052$.

[5] G.D. Ferreira, A.S. Bretas and G. Cardoso. "Optimal distribution protection design considering momentary and sustained reliability indices". In Modern Electric Power Systems. Wroclaw, Poland. September, 2010.

[6] L.G.W. da Silva, R.A.F. Pereira and J.R.S. Mantovani. "Optimized allocation of sectionalizing switches and control and protection devices for reliability indices improvement in distribution systems". In IEEE/PES Transmission \& Distribution Conference \& Exposition: Latin America. November, 2004.

[7] A.M.L. da Silva, A.M. Cassula, L.C. Resende, R.M. Almeida, M.T. Yamasaki, and G.M. Paula. "Reliability evaluation of distribution systems considering automatic reclosers". In 8th International Conference on Probabilistic Methods Applied to Power Systems. Ames, USA. September, 2004.

[8] S. Jamali and H. Shateri. "Optimal siting of recloser and sectionalizers to reduce non-distributed energy". In IEEE/PES Transmission and Distribution Conference \& Exhibition: Asia and Pacific. Dalian, China, 2005.

[9] C.S. Chen, C.H. Lin, H.J. Chuang, C. S. Li, M. Y. Huang and C.W. Huang. "Optimal placement of line switches for distribution automation systems using immune algorithm". IEEE Transactions on Power Systems. Vol. 21, pp. 1209-1217. August, 2006. ISSN: 0885-8950. DOI: 10.1109/TPWRS.2006.876673.

[10] L.G.W. da Silva, R.A.F. Pereira and J.R.S. Mantovani. "Alocação otimizada de dispositivos de controle e proteção em redes de distribuição". Revista Controle \& Automação. Vol. 21, pp. 294-307. Maio, 2010. ISSN: 0103-1759. DOI: 10.1590/ S0103-17592010000300007.

[11] H.H. Dezaki, H.A. Abyaneh, A. Agheli and K. Mazlumi. "Optimized switch allocation to improve the restoration energy in distribution systems". Journal of Electrical Engineering. Vol. 63, pp. 47-52. February, 2012. ISSN: 1335-3632. DOI: $10.2478 /$ v10187-012-0007-9.

[12] J. Burke. "Hard to find information about distribution systems", pp. 1-49. 5 de Agosto de 2013. URL: http://www05.abb.com/ global/scot/scot235.nsf/veritydisplay/91a d3a29a50978bf85256c550053db0d/\$file/ hard.to.find.6th.pdf

[13] P.M. Anderson. "Power System Protection". A John Wiley \& Sons, Inc., Publication. Vol. 4, pp. 41-95. New York, USA. ISBN: 0-7803-3427-2. 1999. 
[14] P.M.S. Carvalho, L.A.F.M. Ferreira and A.J.C. da Silva. "A decomposition approach to optimal remote controlled switch allocation in distribution systems". IEEE Transactions on Power Systems. Vol. 20, pp. 1031-1036. April, 2005. ISSN: 0885-8977. DOI: 10.1109/ TPWRD.2004.838470.

[15] I.W.G. on System Desing. "IEEE Guide for Electric Power Distribution Reliability Indices, IEEE Std 1366-2003 (Revision of IEEE Std 1366-1998)". The Institute of Electrical and Electronics Engineers, Inc, pp. 1-35. New York, USA. ISSN: 978-07381-3890-9. 2004.

[16] L. Goel and R. Billinton. "Evaluation of interrupted energy assessment rates in distribution systems". IEEE Transactions on Power Delivery. Vol. 4, pp. 1876-1882. October, 1991. ISBN: 0885-8977. DOI: 10.1109/61.97735.

[17] K. Deb. "Multi-Objective Optimization Using Evolutionary Algorithms". John Wiley \& Sons, LTD, pp. 239-286. New York, USA. ISBN: 978-0-471-87339-6. 2000.

[18] K. Deb, A. Pratap, S. Agarwal and T. Meyarivan. "A fast and elitist multi-objective genetic algorithm: NSGA-II". IEEE Transactions on evolutionary computations. Vol. 6, pp. 182-197. April, 2002. ISSN: 1089-778X. DOI: 10.1109/4235.996017.

[19] D. Shirmoharmnadi, H. Hong, A. Semlyen and G. Luo. "A compensation-based power flow method for weakly meshed distribution and transmission networks". IEEE Transactions on Power Systems. Vol. 3, pp. 753-762. May, 1988. ISSN: 0885-8950. DOI: $10.1109 / 59.192932$.

[20] C.A.C. Coello, D.A.V. Veldhuizen and G.B. Lamont. "Evolutionary Algorithms for Solving Multi-Objective Problems". Kluwer Academic Publishers, pp. 59-179 New York, USA. ISBN: 0-306-46762-3. 2002.

[21] S. Luke. Essentials of Metaheuristics, G.M. University. $2^{\text {a }}$ Edition, pp. 133-171. USA. ISBN: 978-1-300-54962-8. 2013.

[22] I.J. Ramirez-Rosado and J.L. Bernal-Agustin. "Genetic algorithms applied to the design of large power distribution systems". IEEE Transactions on Power Systems. Vol. 13, pp. 696-703. May, 1998. ISSN: 0885-8950. DOI: $10.1109 / 59.667402$. 